\title{
The efficacy and safety of Oramorph analgesia post paediatric tonsillectomy or adenotonsillectomy- a prospective service evaluation
}

\author{
Tharsika Myuran 1,2, Laura Harding' ${ }^{2}$, Leon Fletcher ${ }^{2}$, Mark Puvanendran ${ }^{2}$ \\ 1. Basildon and Thurrock University Hospitals NHS Foundation Trust, 2. Mid Essex Hospitals NHS Trust
}

\section{INTRODUCTION}

Oramorph prescribing is recommended as an alternative to codeine in the paediatric population undergoing (adeno)tonsillectomy[1]. Following pilot audit, children in Mid Essex Hospitals NHS Trust receive a schedule for over-the-counter analgesia with a backup of Oramorph. We aimed to assess the safety and efficacy of Oramorph analgesia post paediatric (adeno)tonsillectomy.

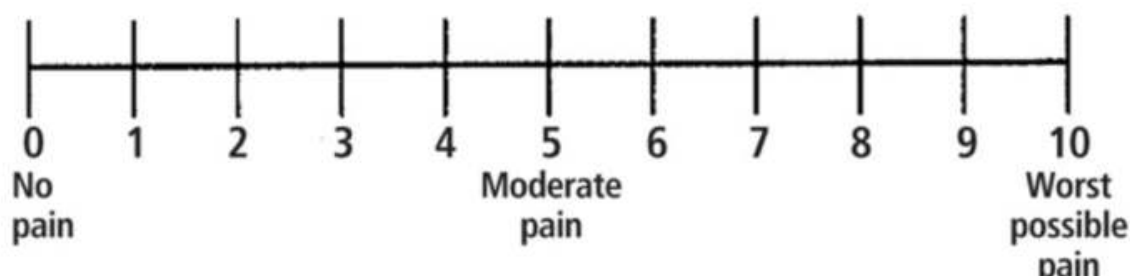

Wong-Baker FACES Pain Rating Scale

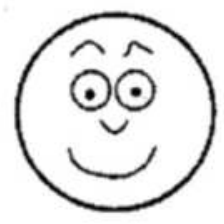

0 NO HURT

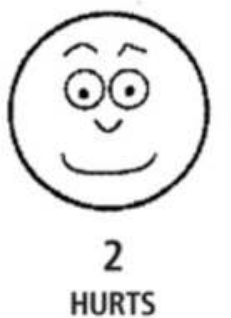

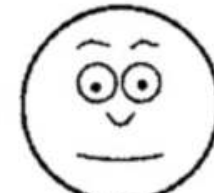

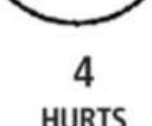

LITTLE MORE
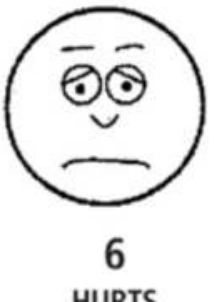

HURTS

EVEN MORE
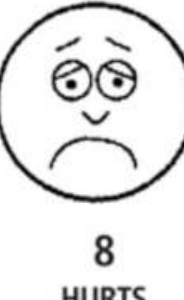

HURTS
WHOLE LOT

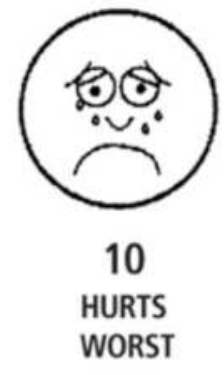

\section{METHODS}

Paediatric patients undergoing

(adeno)tonsillectomy were prospectively audited for: indication, operation, surgeon, anaesthetist, operative technique, peri/post-op analgesia use, nausea, vomiting and pain control using the modified Wong-Baker tool[2] on day $3 / 5^{*}, 7,14$ and a further phonecall on day 20 to note any GP contact, readmission rate, complications. All patients were given advice to take regular and spaced apart paracetamol and ibuprofen, and to use Oramorph for breakthrough analgesia.

*first phonecall would be on day 5 if day 3 was a weekend

\section{RESULTS}

Oramorph $0.2 \mathrm{mg} / \mathrm{kg} 4-6$ hourly PRN max TDS is routinely prescribed $(n=39,100 \%)$. 3 patients were uncontactable post op therefore excluded from the analyses.

Pain scores were not significantly different by operation, indication, surgeon, anaesthetist, operative technique, pre/peri op analgesia. $93 \%$ of patients were happy with pain relief given.

19 patients (52\%) were using Oramorph as part of their analgesic regime in the immediate post-op period, where three had nausea and one had vomiting, none requiring extra input.

There were no significant differences in pain scores between those taking Oramorph and those not taking Oramorph (fig.1). Patients who took Oramorph had higher percentages of normal eating, however this did not reach significance $p>0.05$ (fig 2).

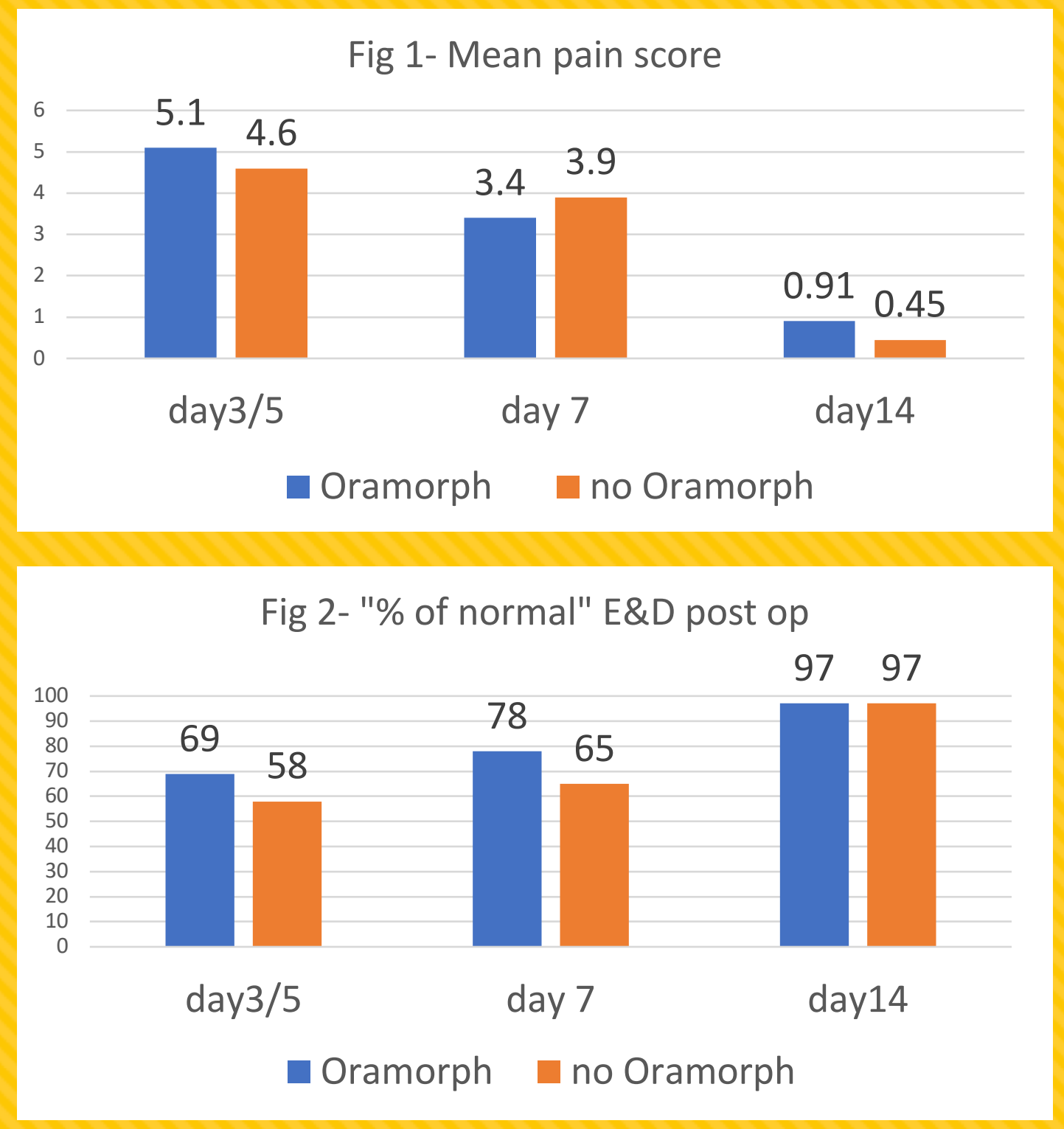

